\title{
Hydro-osmotic Instabilities in Active Membrane Tubes
}

\author{
Sami C. Al-Izzi, ${ }^{1,2,3,4}$ George Rowlands, ${ }^{2}$ Pierre Sens, ${ }^{3,4}$ and Matthew S. Turner ${ }^{2,5}$ \\ ${ }^{1}$ Department of Mathematics, University of Warwick, Coventry CV4 7AL, United Kingdom \\ ${ }^{2}$ Department of Physics, University of Warwick, Coventry CV4 7AL, United Kingdom \\ ${ }^{3}$ Institut Curie, PSL Research University, CNRS, Physical Chemistry Curie, F-75005 Paris, France \\ ${ }^{4}$ Sorbonne Universités, UPMC Univ Paris 06, CNRS, UMR 168, F-75005 Paris, France \\ ${ }^{5}$ Centre for Complexity Science, University of Warwick, Coventry CV4 7AL, United Kingdom
}

(Received 1 August 2017; published 30 March 2018)

\begin{abstract}
We study a membrane tube with unidirectional ion pumps driving an osmotic pressure difference. A pressure-driven peristaltic instability is identified, qualitatively distinct from similar tension-driven Rayleigh-type instabilities on membrane tubes. We discuss how this instability could be related to the function and biogenesis of membrane bound organelles, in particular, the contractile vacuole complex. The unusually long natural wavelength of this instability is in agreement with that observed in cells.
\end{abstract}

DOI: 10.1103/PhysRevLett.120.138102

The "blueprint" for internal structures in living cells is genetically encoded but their spatiotemporal organization ultimately relies on physical mechanisms.

A key contemporary challenge in cellular biophysics is to understand the physical self-organization and regulation of organelles [1,2]. Eukaryotic organelles bound by lipid membranes perform a variety of mechanical and chemical functions inside the cell and range in size, construction, and complexity [3]. A quantitative understanding of how such membrane bound organelles function have applications in bioengineering, synthetic biology, and medicine. Most models of the shape regulation of membrane bound organelles invoke local driving forces, e.g., membrane proteins that alter the morphology (often curvature) [4-6]. However, other mechanisms, such as osmotic pressure, could play an important role [7].

Membrane tubes are ubiquitous in cells, being found in organelles such as the Golgi and endoplasmic reticulum [3] and elsewhere. Models for their formation typically involve the spontaneous curvature of membrane proteins [5] or forces arising from molecular motors, attached to the membrane, that pull tubular tethers as they move along microtubules [8]. Many of these tubules may contain transmembrane proteins that can alter the osmotic pressure by active transport of ions. Most work on the biogenesis of cellular organelles has focused on their static morphology and generally not on their nonequilibrium dynamics. In what follows, we consider an example in which the out-of-

Published by the American Physical Society under the terms of the Creative Commons Attribution 4.0 International license. Further distribution of this work must maintain attribution to the author(s) and the published article's title, journal citation, and DOI. equilibrium dynamics drives the morphology (Fig. 1). Our study is inspired by the biophysics of an organelle called the contractile vacuole complex but additionally reveals a new class of instabilities not previously studied that are of broad, perhaps even universal, physiological relevance.

The contractile vacuole complex (CVC) is an organelle found in most freshwater protists and algae that regulates osmotic pressure by expelling excess water [10-14]. Its primary features is a main vesicle $(\mathrm{CV})$ that is inflated by osmosis and periodically expels its contents through the opening of a large pore-probably in response to membrane tension-connecting it to the extracellular environment, thereby regulating cell volume $[9,14]$. Water influx into

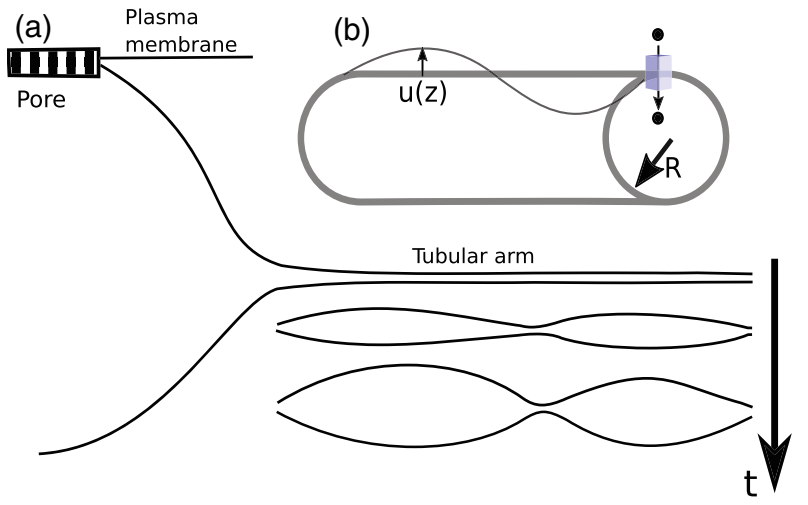

FIG. 1. (a) Diagram of the contractile vacuole complex. The tube is shown connected to the main body of the CV (left). As ions are pumped in, increasing the osmotic pressure, the tube undergoes a swelling instability and undulations develop with some wavelength $\lambda$. This phenomena is observed in the contractile vacuoles of, e.g., Paramecium multimicronucleatum $[9,10]$. (b) Schematic of a membrane tube with ion pumps and surface undulations. An illustration of a representative ion pump is shown in the top right. 
the CVC is due to an osmotic gradient generated by adenosine-triphosphate-hydrolyzing proton pumps in the membrane that move protons into the CVC [12,15-17]. In many organisms, such as Paramecium multimicronucleatum, the CVC includes several membrane tubular arms connected to the main vesicles, which are thought to be associated with the primary sites of proton pumping and water influx activity [18]. The tubular arms do not swell homogeneously in response to water influx, but rather show large undulatory bulges with a size comparable to the size of the main $\mathrm{CV}$, leading us to speculate that this might even play a role in $\mathrm{CV}$ formation de novo. These tubular arms appear to be undergoing a process similar to the Pearling or Rayleigh instability of a membrane tube under high tension [19-26] or an axon under osmotic shock [27], but with a much longer natural wavelength: Rayleigh instabilities have a natural wave length $\lambda \sim R$ where $R$ is the tube radius. Here we derive the dynamical evolution of a membrane tube driven out of equilibrium by osmotic pumping.

In the CVC, the tubular arms are surrounded by a membrane structure resembling a bicontinuous phase made up of a labyrinth tubular network called the smooth spongiome. We assume this to represent a reservoir of membrane keeping membrane tension constant and uniform during tube inflation. It is possible to implement more realistic area-tension relations [26]; however, this is beyond the scope of the present work.

The CVC is comprised of a phospholipid bilayer membrane. This bilayer behaves in an elastic manner $[28,29]$. At physiological temperatures these lipids are in the fluid phase $[3,29]$. For simplicity, we will treat the bilayer as a purely elastic, fluid membrane in the constant tension regime, neglecting the separate dynamics of each leaflet. The membrane free energy involves the mean curvature $H$ and surface tension $\gamma[28,30,31]$

$$
\mathcal{F}=\int_{\mathcal{S}} d A\left(\frac{\kappa}{2}(2 H)^{2}+\gamma\right)-\int \Delta P d V
$$

where $d A$ and $d V$ are the area and volume elements on $\mathcal{S}, \kappa$ is the bending rigidity, and $\Delta P$ is the pressure difference between the fluid inside and outside the tube. Assuming radial symmetry and integrating over the volume of the tube, we obtain

$$
\begin{aligned}
\mathcal{F}= & 2 \pi \int_{-\infty}^{\infty} d z\left[\frac{\kappa}{2} r \frac{1}{\sqrt{1+\left(\partial_{z} r\right)^{2}}}\left(\frac{\partial_{z z} r}{1+\left(\partial_{z} r\right)^{2}}-\frac{1}{r}\right)^{2}\right. \\
& \left.+\gamma r \sqrt{1+\left(\partial_{z} r\right)^{2}}-\frac{1}{2} r^{2} \Delta P\right]
\end{aligned}
$$

where $r(z, t)$ is the radial distance of the axisymmetric membrane from the cylindrical symmetry axis and $z$ measures the coordinate along that axis (see Supplemental Material [32] for details).
We use Eq. (2) as a model for the free energy of a radial arm of the CVC. Ion pumps create an osmotic pressure difference that drive a flux of water to permeate through the membrane. We calculate the dominant mode of the hydroosmotic instability resulting from the volume increase of the tube lumen. We write the radius of the tube as $r(z, t)=R+u(z, t)$, with $u$ assumed small, and make use of the Fourier representation $u(z, t)=\sum_{q} \bar{u}_{q} e^{i q z}$. Absorbing the $q=0$ mode into $R=R(t)$ allows us to write $\int u d z=0$. The free energy per unit length can be written at leading order as

$$
\mathcal{F}=\mathcal{F}^{(0)}+\frac{\pi}{R} \sum_{q} \alpha(q)\left|\bar{u}_{q}\right|^{2},
$$

where

$\alpha(q)=\frac{\kappa}{R^{2}}\left((q R)^{4}-\frac{(q R)^{2}}{2}+1\right)+\gamma(q R)^{2}-\Delta P R$

and

$$
\mathcal{F}^{(0)}=2 \pi\left(\frac{\kappa}{2 R}+\gamma R-\frac{1}{2} \Delta P R^{2}\right) .
$$

Identifying the static pressure difference $\Delta P$ with the Laplace pressure $P_{L}=-\kappa /\left(2 R^{3}\right)+\gamma / R$, the point at which the $q=0$ mode goes unstable can be identified: the membrane tube is unstable for tube radii $R>\sqrt{3} R_{\text {eq }}$ where $R_{\text {eq }}=\sqrt{\kappa / 2 \gamma}$ is the equilibrium radius of a tube with $\Delta P=0$. This criterion for the onset of the instability is the same as the Rayleigh instability on a membrane tube [24]; however, the instability is now driven by pressure not surface tension. This is a crucial difference. It leads to a qualitatively different evolution of the instability, as we now show. In what follows we are interested in the dynamics of the growth of unstable modes after the cylinder has reached radius $\sqrt{3} R_{\text {eq }}$. Our initial condition is a tube under zero net pressure, although the choice of initial condition is not crucial. We assume that the number of proton pumps moving ions from the cytosol into the tubular arm depends only on the initial surface area; i.e., it is fixed as the tube volume (and surface) varies.

We denote the number of ions per unit length in the tube as $n$ and write an equation for the growth of $n$ as

$$
\frac{d n}{d t}= \begin{cases}0, & t \in(-\infty, 0) \\ 2 \pi \beta R_{\mathrm{eq}}, & t \in[0, \infty),\end{cases}
$$

where $\beta$ is a constant equal to the pumping rate of a single pump multiplied by the initial area density of pumps.

The density of ions $\rho_{I}$ can be obtained by solving Eq. (6) and dividing by volume per unit length $v(t)$, 


$$
\rho_{I}=\frac{n(t)}{v(t)}=\frac{n_{0}}{v(t)}+\frac{2 \pi \beta R_{\mathrm{eq}} t}{v(t)} .
$$

The growth of the tube radius is driven by a difference between osmotic and Laplace pressure [33]. This means the rate equation for the increase in volume can be written in terms of the membrane permeability to water. Assuming that the water permeability (number of water-permeable pores) is constant during tube inflation, we write the volume permeability per unit tube length $\mu^{\prime}=2 \pi R_{\text {eq }} \mu$, where $\mu$ is the (initial) permeability of the membrane. Thus,

$$
\frac{d v}{d t}=\mu^{\prime}\left\{k_{B} T\left[\rho_{I}-\rho_{I}(t=0)\right]-P\right\},
$$

where the osmotic pressure is approximated by an ideal gas law. This can be transformed into an equation for $R(t)$ on the time interval $t \in[0, \infty)$. We identify $P$ with the Laplace pressure. This leads to

$$
\frac{d \tilde{R}}{d \tilde{t}}=\frac{\tau_{\text {pump }}}{\tau_{\mu}} \frac{1}{\tilde{R}}\left[\frac{\tilde{t}}{\tilde{R}^{2}}+\left(1+\frac{\tilde{\gamma}}{\tilde{R}}\right)\left(\frac{1}{\tilde{R}^{2}}-1\right)\right],
$$

where $\tilde{\gamma}=\left\{\gamma /\left[k_{B} T R_{\text {eq }} \rho_{I}(t=0)\right]\right\}, \quad \tau_{\text {pump }}=\left\{\left[R_{\text {eq }} \rho_{I}(t=0)\right] /\right.$ $2 \beta\}, \quad \tilde{t}=\left(t / \tau_{\text {pump }}\right), \quad \tilde{R}=\left(R / R_{\mathrm{eq}}\right), \quad$ and $\quad \tau_{\mu}=\left\{R_{\mathrm{eq}} /\right.$ $\left.\left[\mu^{\prime} k_{B} T \rho_{I}(t=0)\right]\right\} . \tau_{\text {pump }}$ and $\tau_{\mu}$ represent the time scales of pumping and permeation of water, respectively. The experimental time scale for radial arm inflation is consistent with a value of $\tau_{\text {pump }} \sim 1-10^{-1} \mathrm{~s}$. These dynamics assume our ensemble conserves surface tension, not volume (as in the usual Rayleigh instability). This proves to be a crucial difference.

Values of $R_{\mathrm{eq}}=25 \mathrm{~nm}, \gamma=10^{-4} \mathrm{~N} \mathrm{~m}^{-1}$, and hence $\kappa$ are estimated using experimentally measured values from $[34,35]$. We take a typical ionic concentration in the cytosol of a protist for $\rho_{I}(t=0)=3.0 \times 10^{8} \mu \mathrm{m}^{-3}$ (around $10 \mathrm{mMol})[12,29,36]$. Making an order of magnitude estimate of $\beta$ from the literature on the $\operatorname{CVC}[12,37,38]$ leads to estimates of $\beta \sim 10^{6}-10^{9} \mu \mathrm{m}^{-2} \mathrm{~s}^{-1}$. Temperature is taken as $T=310 \mathrm{~K}$. The permeability of polyunsaturated lipid membranes is thought to be around $\mu=$ $10^{-4} \mu \mathrm{m} \mathrm{Pa}^{-1} \mathrm{~s}^{-1}$ [39]. This permeability could be much larger in the presence of water channels, but we find that our results are rather insensitive to increasing the value of $\mu$ because, for physiological parameter values, our model remains in the rapid permeation regime, i.e., $\tau_{\mu} / \tau_{\text {pump }} \ll 1$. This permits a multiple time scales expansion [40] of Eq. (9). With $\tilde{\gamma} \sim 10^{-3} \ll 1$, we find the approximate asymptotic solution

$$
\tilde{R}(t)=\left(\frac{t}{\tau_{\text {pump }}}+1\right)^{1 / 2}+\mathcal{O}\left(\frac{\tau_{\mu}}{\tau_{\text {pump }}}\right) .
$$

This solution agrees well with numerical solutions to Eq. (9). Using Eqs. (10) and (4), we can compute the time at which each $q$ mode goes unstable (see Supplemental Material [32]).

We now proceed to derive the dynamical equations for the Fourier modes. The equations governing the solvent flow are just the standard inertia free fluid equations for velocity field $\vec{v}$. These are the continuity and Stokes equations for incompressible flow

$$
\vec{\nabla} \cdot \vec{v}=0 ; \quad \vec{\nabla} P=\eta \nabla^{2} \vec{v},
$$

where $P$ is the hydrodynamic pressure and $\eta=10^{-3} \mathrm{~Pa}$ s is the viscosity. The linearized boundary conditions are $\left.v_{r}\right|_{r=R}=\dot{u}+v_{p}$, where $v_{p}$ is the permeation velocity (proportional to the hydrodynamic pressure jump across the membrane: $\left.v_{p}=\left.\mu \Delta P\right|_{r=R}\right)$, and $\left.v_{z}\right|_{r=R}=0$. The second condition is justified by invoking the membrane reservoir as a mechanism for area exchange.

Solving these equations and substituting into the membrane force balance equation gives (in the small $q R$ limit)

$$
\bar{u}_{q}=-\alpha_{L}(q)\left(\frac{q^{2} R(t)}{8 \eta}+\frac{2 \mu R(t)}{R_{\mathrm{eq}}^{3}}\right) \bar{u}_{q},
$$

where $\bar{u}_{q}$ is the Fourier representation of $u$ in the $z$ direction (see Supplemental Material [32] for details). The response function $\alpha_{L}$ is obtained by replacing the static pressure difference by the Laplace pressure $P_{L}$ in Eq. (4). Note that the term involving $\mu$, capturing mode growth due to permeation, is only relevant for wavelengths $\lambda>100 R_{\text {eq }}$; hence, we will discard it in our analysis for simplicity (but retain it in the numerics, for completeness). The growth rate for a given mode is now time dependent; hence, the mode amplitude cannot be obtained from the maximum of the growth rate, but depends on the growth history and must be obtained by solving the full time-dependent problem. We identify the instability as being fully developed when our linearized theory breaks down. We defined the dominant mode of the instability, called $\hat{q}$, as the first mode with an amplitude reaching $\sqrt{\left\langle\left|\bar{u}_{\hat{q}}\right|^{2}\right\rangle}=R_{\text {eq }}$ (a choice that does not influence our results; see Supplemental Material [32]). This occurs at $t=t_{\text {final }}$.

The fluctuations of modes with wave number $q$ about the radius $R(t)$ follow the dynamics of the Langevin equation based on Eq. (12)

$$
\eta(q) \bar{u}_{q}=-\alpha_{L}(q) \bar{u}_{q}+\zeta_{q},
$$

where $\eta(q)=\left(8 \eta / R q^{2}\right)$ and $\zeta_{q}$, the thermal noise, has the following statistical properties

$$
\begin{gathered}
\left\langle\zeta_{q}\right\rangle=0 \\
\left\langle\zeta_{q}\left(t_{1}\right) \zeta_{q^{\prime}}\left(t_{2}\right)\right\rangle=\delta_{q q^{\prime}} \delta\left(t_{1}-t_{2}\right) \frac{k_{B} T R}{\pi \eta(q)} .
\end{gathered}
$$


Here the thermal noise is found using the equipartition theorem and is thus integrated around the tube radius.

Solving this Langevin equation for $\left\langle\left|\bar{u}_{q}\right|^{2}\right\rangle$, using an initial condition of an equilibrium tube and the approximate form of $\tilde{R}(t)$ [Eq. (10)] we find an integral equation for the mode growth

$$
\begin{aligned}
\frac{\left\langle\left|\bar{u}_{q}\right|^{2}\right\rangle}{R_{\mathrm{eq}}^{2}}= & \frac{k_{B} T}{2 \kappa \pi\left(1+\tilde{q}^{4}\right)} e^{[F(0)-F(\tilde{t})]} \\
& +e^{-F(\tilde{t})} \int_{0}^{\tilde{t}} \frac{k_{B} T \tilde{q}^{2}\left(\tilde{t}^{\prime}+1\right)}{\kappa \pi} \frac{\tau_{\mathrm{pump}}}{\tau_{\eta}} e^{F\left(\tilde{t}^{\prime}\right)} d \tilde{t}^{\prime},
\end{aligned}
$$

where $t^{\prime}$ is a time variable integrating over the noise kernel (in units of $\tau_{\text {pump }}$ ), $\tau_{\eta}=8 R_{\text {eq }}^{3} \eta / \kappa, \tilde{q}=q R_{\text {eq }}$, and

$$
\begin{aligned}
F(t)= & \frac{2 \tau_{\text {pump }} \tilde{q}^{2} \tilde{R}(t)}{15 \tau_{\eta}} \\
& \times\left\{40-5 \tilde{t}+\tilde{q}^{2} \tilde{R}(t)^{2}\left[3 \tilde{t}-2+6 \tilde{q}^{2} \tilde{R}(t)^{2}\right]\right\} .
\end{aligned}
$$

Integrating this numerically, together with Eq. (10), we can find the dynamics of the modes. The distribution of mode amplitude against $q$ is shown in Fig. 2. Although the smallest $q$ modes go unstable first, they have very slow growth, and so the mode that dominates the instability arises from the balance between going unstable early (favoring low $q$ ) and growing fast (favoring higher values of $q$ ).

We can compute numerically the natural wavelength associated with the dominant mode $\hat{q}$, the first to reach $\sqrt{\left\langle\left|\bar{u}_{q}\right|^{2}\right\rangle}=R_{\text {eq }}$ (see Fig. 3). This gives a dominant

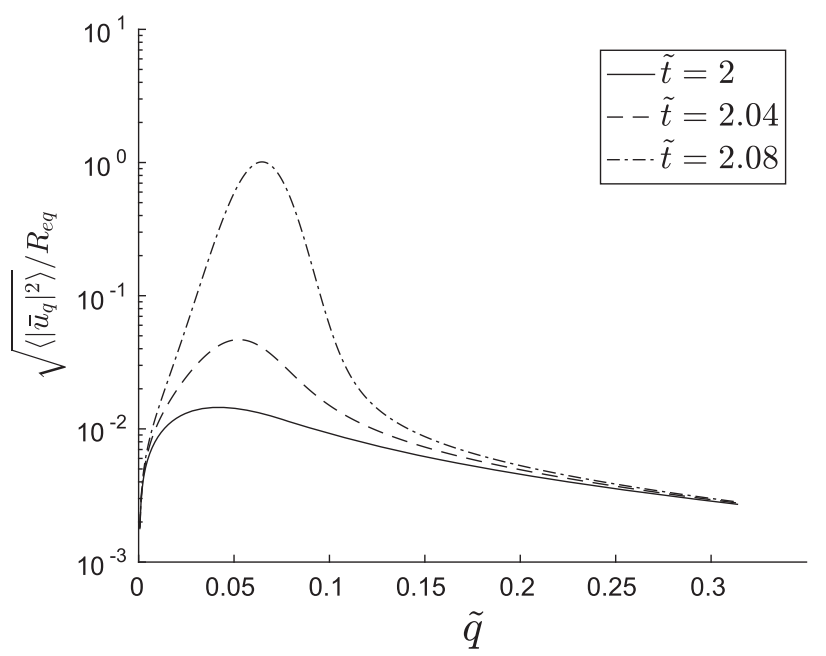

FIG. 2. Plot of the distribution of mode amplitude $\sqrt{\left\langle\bar{u}_{q}^{2}\right\rangle}$ against scaled wave number $\tilde{q}=q R_{\mathrm{eq}}$ for $\tilde{t}=2.0$ (solid), 2.04 (dashed), and 2.08 (dash dotted, the time when the first mode reaches $\left.\sqrt{\left\langle\bar{u}_{q}^{2}\right\rangle}=R_{\text {eq }}\right), \tau_{\eta} / \tau_{\text {pump }} \sim 10^{-6} . R_{\text {eq }}=25 \mathrm{~nm}$, $\gamma=10^{-4} \mathrm{~N} \mathrm{~m}^{-1}$, and $\rho_{I}(t=0)=3.0 \times 10^{8} \mu \mathrm{m}^{-3}$. wavelength $\lambda \sim 100 R_{\text {eq }} \sim 2 \mu \mathrm{m}$ for parameters consistent with the CVC, much larger than that found in the Rayleigh instability, but consistent with observations of the CVC [10]. Understanding why this is the case is not straightforward by inspection of the growth equation (16), but is more easily done by considering the time-dependent growth rate in Eq. (12) (graphically presented in the Supplemental Material [32], Fig. S2). Indeed, at the time $t=t_{\text {final }}$, the dominant mode $\hat{q}$ whose amplitude reaches $\sqrt{\left\langle\left|\bar{u}_{\hat{q}}\right|^{2}\right\rangle}=$ $R_{\text {eq }}$ is very close in value to the fastest growing mode (the peak of the instantaneous growth rate) at that particular time, written $q^{*}$, which can be derived analytically as a function of the tube radius from Eq. (12). As a result of the quasistatic driving of the instability by the ion pumps, the final radius is always only marginally above the critical radius $\sqrt{3} R_{\text {eq }}$ (see Supplemental Material [32]). This is the main factor contributing to the long wavelength, small $q$ instability. In this regime, the fastest growing mode is given by $\tilde{q}^{*} \equiv R_{\text {eq }} q^{*}=\left\{1 /\left[\sqrt{2}(3)^{1 / 4}\right]\right\}\left[\tilde{R}\left(t_{\text {final }}\right)-\sqrt{3}\right]^{1 / 2}$ to leading order (see Supplemental Material [32]). While a qualitatively similar regime exists for tension-driven instabilities, it is only valid very close to the instability threshold and its observation would require a very precise tuning of the tension. Far from threshold, the Rayleigh or Pearling instability shows a universal relationship $\tilde{q}^{*} \sim 0.6 R_{\text {eq }}$ [21-23,26].

A related instability is that of a membrane tube under osmotic shock (see Supplemental Material [32]), for which one finds the most unstable mode to be $\tilde{q}^{*} \sim 0.2$. The difference between the Rayleigh and osmotic shock instabilities is due to the growth rate having a different response when driven by a volume change compared to surface tension (see Supplemental Material [32] for details). The

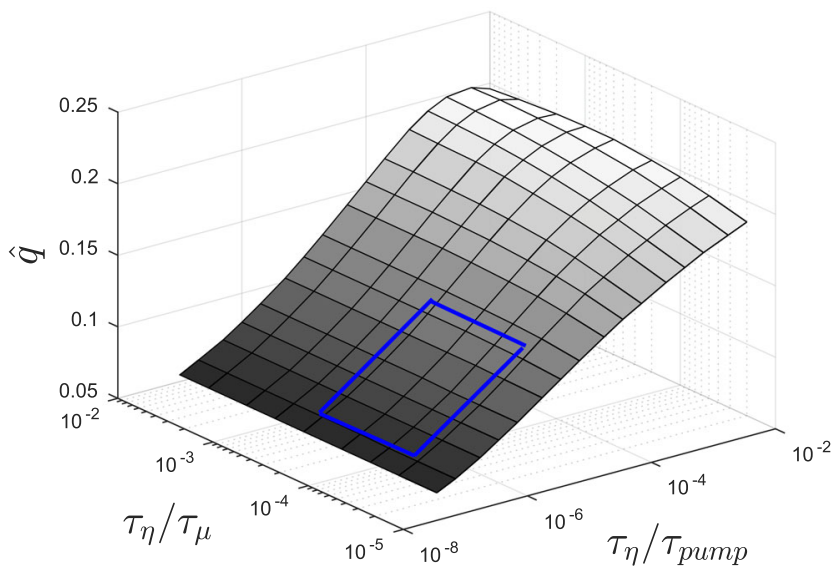

FIG. 3. Plot of dominant wave number $\hat{q}=q^{*} R_{\text {eq }}$ of the instability against ratio of viscous to pumping time scales $\tau_{\eta} / \tau_{\text {pump }}$ and ratio of viscous to permeable time scales $\tau_{\eta} / \tau_{\mu}$. All other parameters are the same as in Fig. 2. The blue rectangle indicates typical physiological parameters. 
constant volume (Rayleigh) instability might be of limited relevance for the morphological changes of cellular membrane tubes, as cellular membranes typically contain a host of membrane channels, including water channels, which allow fairly rapid water transport across the membrane. The osmotic instability that we analyze here recognizes the presence of active pumps in the organelle membrane, which can drive osmotic changes in the organelle lumen [10]. There is some correspondence between the fast pumping limit in Fig. $3\left(\tau_{\eta} / \tau_{\text {pump }}\right.$ large $)$ and the osmotic shock situation. The instantaneous growth rates have the same dependence in the tube radius, but have different time dependences, as the dynamics of tube inflation is different in both cases. The osmotic shock limit is most likely not physiologically accessible to ion pumps. Crucially, one can see in Fig. 3 that the instability length scale is set by dynamical parameters, most importantly, the ratio of the viscosity and pumping time scales. Varying $\tau_{\eta} / \tau_{\text {pump }}$ has the effect of changing the time scale over which the modes go unstable. It is fortuitous that the dominant wavelength does not depend strongly on the pumping rate (see Supplemental Material [32], Fig. S7), the parameter we can estimate least accurately. This suggests a robustness to the wavelength selection that may have important implications for the CVC's biological function. In the physiologically accessible range of parameters for pumping and permeation, this length scale is much larger than the asymptotic limit for either the Rayleigh instability or the osmotic shock instability.

We have developed a model for a water-permeable membrane containing unidirectional ion pumps. Hydroosmotic instabilities realized in cells may be expected to usually lie in this class. Deriving dynamical equations for a membrane tube, we identify an instability driven by this osmotic imbalance. This has a natural wavelength that is set by dynamical parameters and is significantly longer than a Rayleigh or Pearling instability, but is of the same order as seen in the CVC radial arm. We speculate that this instability may provide a mechanism for biogenesis of the CV from a featureless active tube: bulges in the radial arm are similar in size to the main $\mathrm{CV}$. We will further address the question of this organellogenesis in future work.

Thanks to J. Prost and P. Bassereau (Paris), M. Polin (Warwick), and R. G. Morris (Bangalore) for interesting discussions and insight. Thanks to the reviewers for helpful comments and for alerting us to references $[20,26]$. S. C. A.-I. would like to acknowledge funding supporting this work from EPSRC under Grant No. EP/L015374/1, CDT in Mathematics for Real-World Systems.

[1] C. Mullins, The Biogenesis of Cellular Organelles, 1st ed. (Springer, New York, 2005).
[2] Y.-H. M. Chan and W.F. Marshall, Science 337, 1186 (2012).

[3] B. Alberts, A. Jihnson, J. Lewis, M. Raff, K. Roberts, and P. Walter, Molecular Biology of The Cell, 5th ed. (Garland Science, USA, 2008).

[4] R. Heald and O. Cohen-Fix, Curr. Opin. Cell Biol. 26, 79 (2014).

[5] Y. Shibata, J. Hu, M. M. Kozlov, and T. A. Rapoport, Annu. Rev. Cell Dev. Biol. 25, 329 (2009).

[6] U. Jeleri and N. S. Gov, Phys. Biol. 12, 066022 (2015).

[7] D. Gonzalez-Rodriguez, S. Sart, A. Babataheri, D. Tareste, A. I. Barakat, C. Clanet, and J. Husson, Phys. Rev. Lett. 115, 088102 (2015).

[8] A. Yamada, A. Mamane, J. Lee-Tin-Wah, A. D. Cicco, D. Levy, J.-F. Joanny, E. Coudrier, and P. Bassereau, Nat. Commun. 5, 3624 (2014).

[9] D. J. Patterson, Biol. Rev. Camb. Philos. Soc. 55, 1 (1980).

[10] R. D. Allen, BioEssays 22, 1035 (2000).

[11] K. Komsic-Buchmann, L. Wöstehoff, and B. Becker, Eukaryotic Cell 13, 1421 (2014).

[12] C. Stock, H. K. Grønlien, R. D. Allen, and Y. Naitoh, J. Cell Sci. 115, 2339 (2002).

[13] Y. Naitoh, T. Tominaga, M. Ishida, A. K. Fok, M. Aihara, and R. D. Allen, J. Exp. Biol. 200, 713 (1997).

[14] R. Docampo, V. Jimenez, N. Lander, Z.-H. Li, and S. Niyogi, International Review of Cell and Molecular Biology (Elsevier, New York, 2013), Vol. 305, p. 69.

[15] J. Heuser, Q. Zhu, and M. Clarke, J. Cell Biol. 121, 1311 (1993).

[16] T. Nishi and M. Forgac, Nat. Rev. Mol. Cell Biol. 3, 94 (2002).

[17] A. K. Fok, M. S. Aihara, M. Ishida, K. V. Nolta, T. L. Steck, and R. D. Allen, J. Cell Sci. 108, 3163 (1995).

[18] T. Tominaga, R. Allen, and Y. Naitoh, J. Exp. Biol. 201, 451 (1998).

[19] Lord Rayleigh, Philos. Mag. 34, 145 (1892).

[20] S. Tomotika, Proc. R. Soc. A 150, 322 (1935).

[21] T. R. Powers and R. E. Goldstein, Phys. Rev. Lett. 78, 2555 (1997).

[22] R. Bar-Ziv and E. Moses, Phys. Rev. Lett. 73, 1392 (1994).

[23] R. Bar-Ziv, T. Tlusty, and E. Moses, Phys. Rev. Lett. 79, 1158 (1997).

[24] K. L. Gurin, V. V. Lebedev, and A. R. Muratov, J. Exp. Theor. Phys. 83, 321 (1996).

[25] P. Nelson, T. Powers, and U. Seifert, Phys. Rev. Lett. 74, 3384 (1995).

[26] G. Boedec, M. Jaeger, and M. Leonetti, J. Fluid Mech. 743, 262 (2014).

[27] P. A. Pullarkat, P. Dommersnes, P. Fernández, J. F. Joanny, and A. Ott, Phys. Rev. Lett. 96, 048104 (2006).

[28] W. Helfrich, Z. Naturforsch. 28C, 693 (1973).

[29] R. Phillips, J. Kondev, J. Theriot, and H. Garcia, Physical Biology of the Cell, 2nd ed. (Garland Science, USA, 2012).

[30] S. A. Safran, Statistical Thermodynamics of Surfaces, Interfaces, and Membranes, 1st ed. (Westview Press, USA, 2003).

[31] D. Nelson, T. Piran, S. Weinberg, M. E. Fisher, S. Leibler, D. Andelman, Y. Kantor, F. David, D. Bertrand, L. Radzihovsky, M. J. Bowick, D. Kroll, and G. Gompper, Statistical 
Mechanics of Membranes and Surfaces, 2nd ed. (World Scientific, Singapore, 2008).

[32] See Supplemental Material at http://link.aps.org/ supplemental/10.1103/PhysRevLett.120.138102 for detailed calculations and additional figures.

[33] M. Chabanon, J. C. Ho, B. Liedberg, A. N. Parikh, and P. Rangamani, Biophys. J. 112, 1682 (2017).

[34] J. Zimmerberg and M. M. Kozlov, Nat. Rev. Mol. Cell Biol. 7, 9 (2006).

[35] G. Koster, M. VanDuijn, B. Hofs, and M. Dogterom, Proc. Natl. Acad. Sci. U.S.A. 100, 15583 (2003).
[36] M. B. Jackson, Molecular and Cellular Biolophysics, 1st ed. (Cambridge University Press, Cambridge, England, 2006).

[37] R. D. Allen and A. K. Fok, J. Protozool 35, 63 (1988).

[38] T. Tani, R. Allen, and Y. Naitoh, J. Exp. Biol. 203, 239 (2000).

[39] K. Olbrich, W. Rawicz, D. Needham, and E. Evans, Biophys. J. 79, 321 (2000).

[40] J. D. Murray, Asymptotic Analysis, 1st ed. (Springer, New York, 1984). 